\section{Microbiome in Sjögren's syndrome: here we are}

Manasson $e t \mathrm{al}^{1}$ wrote an interesting review on what is known with regard to the application of microbiome research for diagnostic and research purposes within autoimmunity and rheumatology. The authors focused this review on the connection between the microbiome and rheumatoid arthritis (RA) as for long it has been presumed that microbiota are contributors to the development and progression of RA. Besides RA, the review also discussed microbiome studies in systemic lupus erythematosus (SLE) and systemic sclerosis. Remarkably, no attention was paid by Manasson et al to the potential involvement of the microbiome in Sjögren's syndrome (SS). SS is the second most prevalent systemic rheumatic autoimmune disease after RA and the potential involvement of the microbiome in SS-patients is yet also extensively reported. The current knowledge on the relationship between the human microbiome and SS reveals possible pathogenic mechanisms and forms a basis for future treatment options through influencing the microbiome. Thus, the current knowledge on the SS-microbiome connection is an important addition to the review of Manasson et al. ${ }^{1}$

Thus far, all studies that compared the gut microbiome of SS patients with healthy or population controls have shown that the composition of the gut microbiome in SS patients differs from controls. ${ }^{2-7}$ Mandl et al $^{3}$ found that severe intestinal dysbiosis (measured with a $16 \mathrm{~S}$ rRNA-based dysbiosis test) was more prevalent in primary SS (pSS) patients (21\%) than in healthy controls (3\%). Importantly, SS patients with severe intestinal dysbiosis had higher disease activity score, higher faecal calprotectin and lower $\mathrm{C} 4$ concentrations in blood samples, suggesting a connection between dysbiosis in the gut microbiome, gut inflammation, systemic inflammation and disease severity. Other studies also reported correlations between gut microbiome parameters and SS disease severity, especially related to dry eye symptoms. ${ }^{25}$

A relatively frequent finding in the gut microbiome of SS patients is a higher relative abundance of phylum Bacteroides, expressed as a lower Firmicutes:Bacteroides ratio, compared with controls. ${ }^{457}$ We have previously shown that the relative abundance of three Bacteroides species (ie, B. vulgatus, B. uniformis and $B$. ovatus) was significantly higher in pSS patients $(\mathrm{n}=39)$ than in population controls $(\mathrm{n}=965) .^{4}$ Similar to patients with SS, SLE patients also showed a lower Firmicutes:Bacteroides ratio in the gut microbiome. ${ }^{48}$ The three aforementioned Bacteroides species were also higher in SLE patients than in population controls. ${ }^{4}$ Another Bacteroides species, Bacteroides thetaiotaomicron (B. theta), was higher in pSS and SLE patients compared with controls, but only significant for SLE. ${ }^{4}$ The species $B$. theta is of major interest, because Greiling $e t a l^{9}$ identified this species as a potential gut pathobiont (ie, a potential pathogenic micro-organism, which, under normal circumstances, is harmless). Its pathogenic role could be ascribed to the finding that lysates of $B$. theta can bind to serum from anti-Ro60-positive SLE patients. Furthermore, B-cell and T-cell responses to the Ro60-protein occurred after monocolonisation of mice with $B$. theta, subsequently leading to enhanced lupus-like disease in mice. ${ }^{9}$ Because anti-Ro60 autoantibodies are observed in up to $70 \%$ of pSS patients, the findings of Greiling et al may suggest a potential role for this species in the pathogenesis both for SLE and also for pSS. However, there is no evidence for an association between the presence of anti-Ro60 auto-antibodies in serum and $B$. theta relative abundance in faecal samples of both type of patients. ${ }^{49}$ Therefore, future studies should both measure relative abundance and also perform quantitative microbiome profiling. ${ }^{10}$

The Firmicutes:Bacteroides ratio as well as the overall gut microbiota composition of pSS patients and SLE patients are very similar and differ from that of population controls. ${ }^{4}$ Dysbiosis may also be present in other systemic autoimmune diseases. Bellocchi et $a l^{6}$ observed that the gut microbiome of patients with systemic autoimmune diseases (SS, SLE, primary antiphosholipid syndrome and undifferentiated connective tissue disease) differed from that of healthy controls, but not between the of the various systemic autoimmune diseases. Possibly, a dysbiosis in the gut microbiome may predispose to systemic inflammatory diseases.

De Paiva $e t \mathrm{al}^{2}$ showed in a mouse model for SS that gut dysbiosis worsens the response to desiccating stress and increased production of T-cell related cytokines in conjunctival epithelium. Zaheer $e \mathrm{al}^{11}$ showed in another mouse model for spontaneous SS (CD25 knock-out mice) that germ-free CD25KO mice developed sooner and more severe dacryoadenitis than conventional housed mice. Furthermore, Zaheer et $a l^{11}$ and Wang et $a l^{12}$ showed in the same mouse model, that a faecal microbiome transplant from conventional mice to mice with SS-like lacrimal keratoconjunctivitis reversed the dry eye phenotype.

Multiple studies have confirmed that the oral microbiome in SS patients significantly differs from that of healthy individuals. ${ }^{213-21}$ Although we cannot exclude the possibility that certain species of bacteria may be involved in the development of SS and that disease-specific factors of SS influence the oral microbiome, the consensus is that reduced salivary secretion is the most important factor in shaping the oral microbiome in SS. ${ }^{15161820}$ For example, the relative abundance of specific microbiota (ie, genera Lactobacillus, Haemophilus and Neisseria) significantly correlated with salivary secretion rate. ${ }^{1516}$

Manasson et $a l^{1}$ provide a useful overview of the dos and don'ts for microbiome research in rheumatic diseases. Based on the studies discussed above, we suggest three additional 'do or don'ts'. First, studies should include more than one rheumatic disease in order to assess whether there is an underlying shared mechanism by which microbiota play a role in the pathogenesis of rheumatic autoimmune diseases. Second, studies should be extended from human case-control studies to intervention studies and other experimental approaches (eg, ex-vivo studies) in order to obtain essential additional knowledge to explain the results. Finally, human microbiome studies should not be restricted to faecal samples alone.

Taco A van der Meulen $\odot,{ }^{1}$ Arjan Vissink, ${ }^{1}$ Hendrika Bootsma, ${ }^{2}$ Fred K L Spijkervet, ${ }^{1}$ Frans G M Kroese ${ }^{2}$

${ }^{1}$ Oral and Maxillofacial Surgery, Universitair Medisch Centrum Groningen, Groningen, The Netherlands

${ }^{2}$ Rheumatology and Clinical Immunology, University Medical Centre Groningen, Groningen, The Netherlands

Correspondence to Taco A van der Meulen, Oral and Maxillofacial Surgery, Universitair Medisch Centrum Groningen, 9713 GZ Groningen, The Netherlands; t.a.van.der.meulen@umcg.nl

Contributors TAvdM, AV and FGMK were involved in the conception and drafting of the article. All authors were involved in revising the article. All authors approved the final version to be submitted for publication.

Funding The authors have not declared a specific grant for this research from any funding agency in the public, commercial or not-for-profit sectors.

Competing interests None declared.

Patient and public involvement Patients and/or the public were not involved in the design, or conduct, or reporting or dissemination plans of this research.

Patient consent for publication Not required. 
Provenance and peer review Not commissioned; internally peer reviewed.

(c) Author(s) (or their employer(s)) 2020. No commercial re-use. See rights and permissions. Published by BMJ.

\section{Check for updates}

To cite van der Meulen TA, Vissink A, Bootsma $H$, et al. Ann Rheum Dis Epub ahead of print: [please include Day Month Year]. doi:10.1136/annrheumdis-2020-218213

Received 8 June 2020

Accepted 10 June 2020

\section{SLinked}

http://dx.doi.org/10.1136/annrheumdis-2020-218327

Ann Rheum Dis 2020;0:1-2. doi:10.1136/annrheumdis-2020-218213

ORCID iD

Taco A van der Meulen http://orcid.org/0000-0002-1640-7855

\section{REFERENCES}

1 Manasson J, Blank RB, Scher JU. The microbiome in rheumatology: where are we and where should we go? Ann Rheum Dis 2020;79:727-33.

2 de Paiva CS, Jones DB, Stern ME, et al. Altered mucosal microbiome diversity and disease severity in Sjögren syndrome. Sci Rep 2016;18:23561.

3 Mandl T, Marsal J, Olsson P, et al. Severe intestinal dysbiosis is prevalent in primary Sjögren's syndrome and is associated with systemic disease activity. Arthritis Res Ther 2017; 19:237.

4 van der Meulen TA, Harmsen HJM, Vila AV, et al. Shared gut, but distinct oral microbiota composition in primary Sjögren's syndrome and systemic lupus erythematosus. J Autoimmun 2019;97:77-87.

5 Moon J, Choi SH, Yoon CH, et al. Gut dysbiosis is prevailing in Sjögren's syndrome and is related to dry eye severity. PLoS One 2020;15:e0229029.
6 Bellocchi C, Fernández-Ochoa Álvaro, Montanelli G, et al. Identification of a shared microbiomic and metabolomic profile in systemic autoimmune diseases. I Clin Med 2019;8:1291.

7 Mendez R, Watane A, Farhangi M, et al. Gut microbial dysbiosis in individuals with Sjögren's syndrome. Microb Cell Fact 2020;19:90.

8 Hevia A, Milani C, López P, et al. Intestinal dysbiosis associated with systemic lupus erythematosus. mBio 2014;5:e01548-14.

9 Greiling TM, Dehner C, Chen X, et al. Commensal orthologs of the human autoantigen Ro60 as triggers of autoimmunity in lupus. Sci Trans/ Med 2018;10:eaan2306.

10 Vandeputte D, Kathagen G, D'hoe K, et al. Quantitative microbiome profiling links gut community variation to microbial load. Nature 2017;551:507-11.

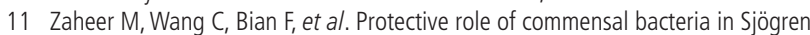
syndrome. J Autoimmun 2018;93:45-56.

12 Wang C, Zaheer M, Bian F, et al. Sjögren-like lacrimal keratoconjunctivitis in germ-free mice. Int J Mol Sci 2018:19:E565.

13 Li M, Zou Y, Jiang Q, et al. A preliminary study of the oral microbiota in Chinese patients with Sjögren's syndrome. Arch Oral Biol 2016:70:143-8.

14 Siddiqui H, Chen T, Aliko A, et al. Microbiological and bioinformatics analysis of primary Sjogren's syndrome patients with normal salivation. J Oral Microbiol 2016;8:31119.

15 van der Meulen TA, Harmsen HJM, Bootsma H, et al. Dysbiosis of the buccal mucosa microbiome in primary Sjögren's syndrome patients. Rheumatology 2018;57:2225-34

16 van der Meulen TA, Harmsen HJM, Bootsma H, et al. Reduced salivary secretion contributes more to changes in the oral microbiome of patients with primary Sjögren's syndrome than underlying disease. Ann Rheum Dis 2018:77:1542-4.

17 Rusthen S, Kristoffersen AK, Young A, et al. Dysbiotic salivary microbiota in dry mouth and primary Sjögren's syndrome patients. PLoS One 2019:14:e0218319.

18 Sembler-Møller ML, Belstrøm D, Locht $\mathrm{H}$, et al. Next-Generation sequencing of whole saliva from patients with primary Sjögren's syndrome and non-Sjögren's sicca reveals comparable salivary microbiota. J Oral Microbiol 2019;11:1660566.

19 Alam J, Lee A, Lee J, et al. Dysbiotic oral microbiota and infected salivary glands in Sjögren's syndrome. PLoS One 2020;15:e0230667.

20 Proctor DM, Fukuyama JA, Loomer PM, et al. A spatial gradient of bacterial diversity in the human oral cavity shaped by salivary flow. Nat Commun 2018:9:681.

21 Sharma D, Sandhya P, Vellarikkal SK, et al. Saliva microbiome in primary Sjögren's syndrome reveals distinct set of disease-associated microbes. Oral Dis 2020;26:295-301. 